\title{
An integrated production-inventory model for deteriorating items to evaluate JIT purchasing alliances
}

\author{
Freddy Pérez ${ }^{a^{*}}$ and Fidel Torres ${ }^{\mathrm{a}}$
}

${ }^{a}$ Department of Industrial Engineering, Universidad de los Andes: Cra $1 N^{\circ} 18 \mathrm{~A}$ 12, Bogotá, Colombia

\begin{tabular}{l}
\hline C H R O N I C L E \\
\hline Article history: \\
Received January 302018 \\
Received in Revised Format \\
February 182018 \\
Accepted May 42018 \\
Available online \\
May 5 2018 \\
\hline Keywords: \\
Inventory model \\
Deterioration item \\
Time value of money \\
Just-in-time purchasing
\end{tabular}

\section{Introduction}

As indicated by $\mathrm{Xu}$ and Chen (2016), just-in-time (JIT) practices have been widely adopted in manufacturing businesses, and for both academics and practitioners, JIT production systems have been recognized as an effective strategy to enhance organizational competitiveness (Chen \& Tan, 2013). In a JIT system, both a vendor and buyer work together in a mutually rewarding long-term partnership to achieve a cost-effective supply chain inventory system. Typically, this is mainly accomplished through the use of lower lot-size and frequent deliveries, and with the correct application of the JIT delivery concept (Matsui, 2007). An extensive literature of empirical studies is available highlighting many principles for adopting JIT, successfully. Readers are encouraged to consult Chen and Tan (2011), Chen and Tan (2013), Negrão et al. (2017), and the references cited therein.

Although, currently, organizations such as Dell, Walmart and many others have earned their success, at least in part, as a result of the JIT management strategy (Michelsen et al., 2014), the ultimate goals of a JIT system, zero-inventories and zero set-up times, are impossible to achieve even in the best JIT-lean applications (Ali et al., 2012; Darlington et al., 2016; Santos et al., 2006). Thus, in these contexts, a common question belonging to the field of inventory theory inexorably arises: what is the optimal

* Corresponding author

E-mail: fa.perez10@uniandes.edu.co (F. Pérez)

2019 Growing Science Ltd.

doi: $10.5267 /$ j.ijiec.2018.5.001

\begin{abstract}
The implementation of just-in-time (JIT) principles has been shown to be worthy of analysis due of adopting JIT management concepts, little work has been accomplished in offering analytical tools for assisting managers for implementing JIT strategy. This paper proposes a new inventory setup multiple-delivery model for deteriorating items by considering the effect of the time value of money (TVM). We propose a solution procedure to determine the optimal decisions that maximize the discounted profit function of this analytical model, and compare it with some other alternatives. Here, we show the derivation of the mathematical model, the algorithm of the
proposed solutions, and the application of the new approach through two numerical experiments. The study reveals that modeling the TVM effect complicates the determination of an optimal JIT inventory policy; nevertheless, we find that accounting for TVM can be decisive in terms of
\end{abstract}

(C) 2019 by the authors; licensee Growing Science, Canada 
smallest lot-size with frequent deliveries that should be used? For a complete discussion regarding the role of lot sizing theory on JIT practices, readers are referred to Andriolo et al. (2014), and Chiarini (2017).

After becoming aware of the need to assist managers in the implementation of some JIT concepts from a mathematical point of view, several researchers conducted studies with this aim. One such area of research, of course, was the modeling of inventory systems under a JIT environment. However, although the large body of empirical studies about JIT systems has demonstrated the great interest from both academics and practitioners in JIT matters, the support of lot sizing theory in JIT practices is still undeveloped.

An economic order quantity (EOQ) model under JIT purchasing agreements was first accounted for by Pan and Liao (1989). However, this model was strongly criticized by Larson (1989) because the delivery cost was set at zero regardless of the number of deliveries scheduled in an order cycle. The total annual operating cost used in the traditional EOQ model was then adapted by Ramasesh (1990) to include the costs associated with small-lot shipments as follows:

$T C=\frac{A D}{Q}+\frac{N F D}{Q}+\frac{H Q}{2 N}$

where $A$ is the cost of placing an order, $D$ is the annual demand, $Q$ is the contract quantity, $N$ is the number of shipments per contract, $F$ is the aggregate cost per shipment, and $H$ is the inventory holding cost per unit per year.

Following the work of Ramasesh (1990), Aderohunmu et al. (1995), Banerjee and Kim (1995), Ha and Kim (1997), and Kim and Ha (2003) addressed the need to model and optimize the costs of both the buyer and vendor simultaneously to operate optimally in a JIT environment. Because a distinctive aspect of the JIT philosophy is to ensure a long-term buyer-vendor relationship based on mutual trust, one of the main findings of these studies was to show mathematically that close co-operation is economically beneficial not just for the buyer but also for the vendor. Banerjee and Kim (1995) stated that such a longterm partnership may be possible if the vendor shares with the buyer the savings resulting from adopting JIT concepts. In this model, the vendor pays the aggregate cost per shipment, and the ordering and holding costs of raw materials are taken into account. Aderohunmu et al. (1995) and Ha and Kim (1997) drew the same conclusion but from the buyer perspective, and excluding raw materials. Kim and Ha (2003), reintroduced the model in Aderohunmu et al. (1995) and Ha and Kim (1997), and found that the optimal delivery size can be unique, that is, without the order quantity and number of deliveries.

Even though the foregoing works made an important contribution by considering an integrated model to successfully implement JIT practices, the impact of deteriorating products on inventory systems was overlooked. Rau et al. (2003) and Lin et al. (2009) incorporated, respectively, a constant deterioration rate into a three and two-echelon supply chain; however, the planning period was assumed to be given to make possible their cost function derivation. In subsequent related studies, Yan et al. (2011) extended Kim and Ha (2003) to address the effects of deterioration, Sarkar (2013) extended the work accomplished by Yan et al. (2011) through employing an algebraic optimization method under different deterioration patterns, and Chang (2014) extended Yan et al. (2011) and Sarkar's (2013) work by providing an improved solution procedure. In these three papers, however, although there was no longer an assumption of a known planning period, the cost functions had to be derived using an analytical geometric and algebraic method instead of a differential calculus-based approach by assuming that items' deterioration was sufficiently small that its squares and higher powers could be neglected. The reason of using this approach, as explained by Yan et al. (2011), is because the inventory level of the supplier changes suddenly and forms inflexions that make it difficult to use the classical optimization techniques. 
The aforementioned issue does not only arise over inventory models developed for deteriorating items but also when neglecting items' deterioration, where, as proved to be the case for deterioration, the derivation of the supplier's average inventory under JIT practices had its own foundation in the mathematical expression derived by Joglekar (1988). Although this expression was initially applied in a different context, it resulted to be particularly suitable for JIT environments. Thus, when it became possible to release the common assumption of a single delivery per order to allow multiple deliveries per order within the same production setting cycle, the discussed new research stream began to be discussed, by emphasizing its applicability to JIT strategic alliances pursuing the operational reduction of set-up times, inventories, and lead times.

In the light of the above, it can be said that important advances have been accomplished regarding accommodating traditional EOQ/EPQ formulas to account for the particularities of JIT systems. However, much more research is still necessary so that all the practical features of real inventory systems under a JIT environment are completely studied and analyzed. Two practical business characteristics included in the present study are the effect of time value of money (TVM) and product deterioration. As argued by White et al. (1999) and many other authors, the objective of JIT purchasing is to improve quality, flexibility and levels of service from suppliers by developing a long-term buyer-vendor coordination based on mutual trust. Thus, the effect of the TVM may be crucial for evaluating and implementing such a long-term partnership, as became apparent in well-known and abundantly used discounted cash flow analyses. Moreover, the incorporation of product deterioration into JIT inventory models is also worth of analysis because many items that belong to different product categories, such as medicine, volatile liquids, blood, and food products, have a deterioration rate that directly has an effect on lot sizing calculation.

As a result, we extent and generalize the works of Yan et al. (2011), Sarkar (2013), and Chang (2014) by introducing a new deteriorating production-inventory model under the TVM to assist JIT partnerships. The major contributions of our work are as follows:

- We model and analyze the TVM effect, which, to the best of our knowledge, has not been conducted in studies on JIT inventory models.

- We use differential calculus to derive cost functions, which are expected to drive future research toward the study and analysis of other inventory characteristics in JIT environments.

- We present and compare five easy to implement algorithms that aim to determine the optimal decisions of the proposed model by exploiting the existence of analytical expressions, in addition to the existence of leading commercial software.

The remainder of this paper is structure as follows: In Section 2, we present notation and assumptions. In Section 3, we introduce the proposed inventory model for deteriorating items under the TVM. In section 4, we describe the solution procedures to measure and maximize the benefit of JIT agreements. In Section 5, we present numerical examples to compare the efficiency of different approaches and provide guidelines for the practical use of the modeling approach presented in this paper. Finally, in Section 6 , we conclude by summarizing the main findings and describing directions for potential future research.

\section{Notation and assumptions}

To simplify the analysis and derivation of the mathematical model, we use the following notation and assumptions.

\subsection{Notation}

$A$ ordering cost (\$/order) 
$C \quad$ setup cost for a production batch (\$/setup)

$C_{B}, C_{S}$ deterioration cost per unit for the buyer and supplier (\$/unit)

$D \quad$ constant demand rate (units/year)

$F \quad$ constant transportation cost per delivery (\$/delivery)

$H \quad$ time planning horizon (years)

$H_{B}, H_{S}$ inventory holding cost for the buyer and supplier (\$/unit/year)

$m \quad$ number of inventory cycles (an integer decision variable) over $[0, H]$

$N \quad$ number of deliveries per inventory cycle: $N \geq 1$ (an integer decision variable)

$P \quad$ production rate (units/year)

$q \quad$ delivery lot-size in units (a controllable parameter: given by $m$ and $N$

$Q_{P} \quad$ production lot-size per cycle: $Q_{P}=P T_{Q}$

$Q_{S} \quad$ supplier inventory at time $T_{Q}$ per cycle (units)

$r \quad$ discount rate (effective per year compounded continuously)

$S \quad$ constant product selling price per unit (\$/unit)

$T$ duration of each inventory cycle in units of time: $T=H / m$

$T_{d} \quad$ length of time between deliveries: $T_{d}=H /(m N)$.

$T_{q} \quad$ supplier length of time in producing $q$ units: $T_{q}=\ln P /(P-\theta q)$

$T_{Q} \quad$ supplier length of time in reaching $Q_{S}$ level

$\tau$ transport time (years)

$u \quad$ variable cost per unit produced (\$/unit)

$V \quad$ unit variable cost for order handling and receiving (\$/unit)

$\theta_{B}, \theta_{S}$ deterioration rate for the buyer and supplier (\%/year)

$Z_{B}, Z_{S}$ present value of the total buyer's and supplier's inventory costs (\$)

$Z_{0} \quad$ present value of JIT investment during $H$

$Z_{T} \quad$ integrated discounted profit (IDP): a function of $m$ and $N$

\subsection{Assumptions}

We make the following assumptions to develop the proposed inventory model for deteriorating items under the TVM.

i. Both a single producer and single buyer are willing to exchange necessary information (e.g., costs, demand, production and inventory records).

ii. Multiple lot-size deliveries per order are considered instead of a single delivery per order. The transportation time for these deliveries is known and constant. Shortages are not allowed.

iii. The producer delivers the same lot-size of finished goods at fixed-time intervals.

iv. A single item is considered over a prescribed period of $H$ units of time.

v. The demand and production rate are constant and deterministic $(P>D)$.

vi. All cost parameters are known and constant.

vii. The buyer pays transportation and other handling costs of frequent deliveries.

viii. The planning horizon $H$ is finite and the effect of the TVM is considered.

\section{Model formulation}

In this section, we consider the effect of the TVM when evaluating JIT purchasing agreements through a single-setup multiple-delivery inventory model for deteriorating items. First, we derive the discounted cost functions for the buyer and supplier, and then we present the IDP function of the JIT partnership together with our proposed optimization problem.

To consider the effect of the TVM, the total time horizon $H$ is divided into $m$ equal parts; hence, each inventory cycle is given by $T=H / m$. When the buyer places an order with the supplier for the quantity 
of finished goods needed in period $T$, the supplier is allowed to deliver $N$ smaller lots of size $q$ over fixed-time intervals equal to $H /(m N)$. Each supplier's inventory cycle $H / m$ can then be divided into two components: $T_{Q}$, the time in which the supplier manufactures finished goods, and $T-T_{Q}$, the time in which the supplier does not produce any products. The pattern followed by the inventory level is illustrated in Fig. 1. Fig. 1 (a) shows the buyer's inventory level, whereas Fig. 1 (b) shows the supplier's inventory level when $N=6$.

\subsection{Buyer's discounted cost function}

Consider the variation of the buyer's inventory between the first and second delivery. This variation occurs because of the combined effect of demand and deterioration. Thus, the variation of the buyer's inventory with respect to time $t, I_{B}(t)$, can be described by the following differential equation:

$$
\frac{d I_{B}(t)}{d t}=-D-\theta_{B} I_{B}(t), \quad T_{q}+\tau \leq t \leq T_{q}+\tau+\frac{H}{m N} .
$$

With the boundary condition $I\left(T_{q}+\tau+T / N\right)=0$, the solution of Eq. (1) can be represented by

$$
I_{B}(t)=\frac{D}{\theta_{B}}\left(e^{\theta_{B}\left(T_{q}+\tau+\frac{H}{m N}-t\right)}-1\right), \quad T_{q}+\tau \leq t \leq T_{q}+\tau+\frac{H}{m N} .
$$

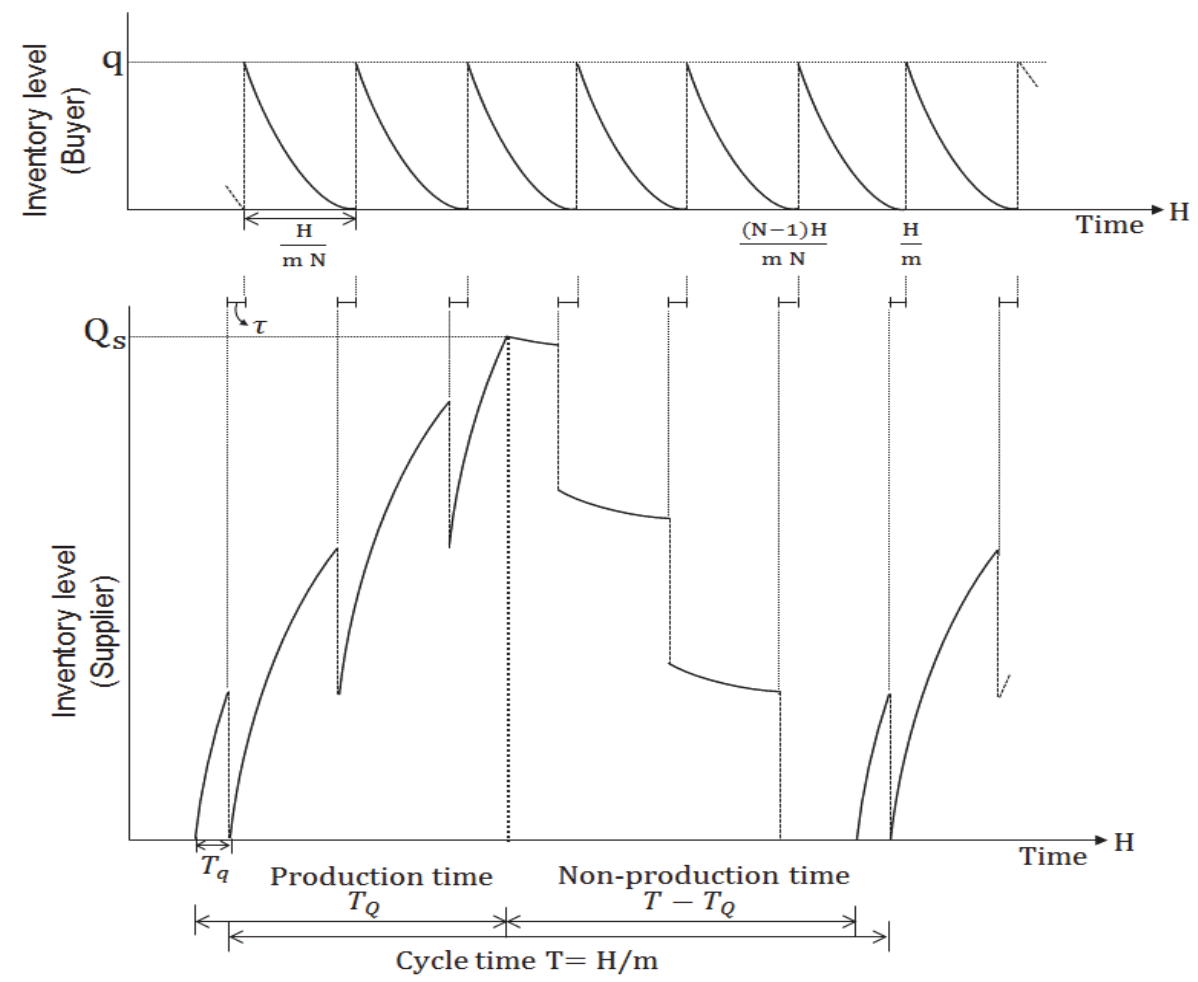

Fig 1. Inventory level versus time for the (a) buyer and (b) supplier

We assumed that the buyer's inventory changes to $q$ units when it receives the first delivery; thus, if we use Eq. (2) at time $t=T_{q}+\tau$, then we obtain the delivery lot-size as follows:

$$
q=\frac{D}{\theta_{B}}\left(e^{\theta_{B}\left(\frac{H}{m N}\right)}-1\right)
$$


Additionally, considering Eq. (2), the present value of the holding costs and disposal costs between the first and second delivery can be written as

$$
\begin{aligned}
P V^{\prime}{ }_{H C_{B}}=P V_{H_{B}}^{\prime} & +P V^{\prime} C_{B}=\left(H_{B}+C_{B} \theta_{B}\right) e^{-r\left(T_{q}+\tau\right)} \int_{T_{q}+\tau}^{T_{q}+\tau+\frac{H}{m N}} I_{B}(t) e^{-r\left(t-\left(T_{q}+\tau\right)\right)} d t \\
& =\left(H_{B}+C_{B} \theta_{B}\right) \int_{T_{q}+\tau}^{T_{q}+\tau+\frac{H}{m N}} I_{B}(t) e^{-r t} d t \\
& =\left(H_{B}+C_{B} \theta_{B}\right) D\left(\frac{e^{\theta_{B} \frac{H}{m N}}-1}{\theta_{B}\left(r+\theta_{B}\right)}+\frac{e^{-r \frac{H}{m N}}-1}{r\left(r+\theta_{B}\right)}\right) e^{-r\left(T_{q}+\tau\right)} .
\end{aligned}
$$

Hence, the present value of the total holding costs and disposal costs during the entire time horizon, denoted by $P V_{H C_{B}}$, is given by

$$
\begin{aligned}
P V_{H C_{B}}=P V_{H_{B}} & +P V_{C_{B}}=\sum_{i=0}^{m N-1}\left(P V_{H_{B}}^{\prime}+P V_{C_{b}}^{\prime}\right) e^{-r(i T)} \\
& =\left(H_{B}+C_{b} \theta_{B}\right) D\left(\frac{e^{\theta_{B} \frac{H}{m N}}-1}{\theta_{B}\left(r+\theta_{B}\right)}+\frac{e^{-r \frac{H}{m N}}-1}{r\left(r+\theta_{B}\right)}\right) e^{-r\left(T_{q}+\tau\right)} \frac{1-e^{-r H}}{1-e^{-r \frac{H}{m N}}} .
\end{aligned}
$$

Because there are $m$ orders and $m \times N$ deliveries in entire time horizon $H$, the present value of total ordering costs $A$, transportation costs $F$, handling costs $V$, and unit costs $u$ is given by

$$
\begin{gathered}
P V_{A}+P V_{F}+P V_{V c}=A \sum_{i=1}^{m} e^{-r\left((i-1) T+T_{q}+\tau\right)}+(F+q(V+u)) \sum_{i=1}^{m N} e^{-r\left((i-1) T+T_{q}+\tau\right)} \\
=e^{-r\left(T_{q}+\tau\right)}\left(A \frac{1-e^{-r H}}{1-e^{-r \frac{H}{m}}}+(F+q(V+u)) \frac{1-e^{-r H}}{1-e^{-r \frac{H}{m N}}}\right)
\end{gathered}
$$

Consequently, the present value of the total buyer cost is

$$
Z_{B}(m, N)=P V_{A}+P V_{F}+P V_{V C}+P V_{H C_{B}}
$$

Thus,

$$
\begin{aligned}
Z_{B}(m, N)=e^{-r\left(T_{q}+\tau\right)}\left[A \frac{1-e^{-r H}}{1-e^{-r \frac{H}{m}}}+\right. \\
\left.\left(F+q(V+u)+\left(H_{B}+C_{B} \theta_{B}\right) D\left(\frac{e^{\theta_{B} \frac{H}{m N}}-1}{\theta_{B}\left(r+\theta_{B}\right)}+\frac{e^{-r \frac{H}{m N}}-1}{r\left(r+\theta_{B}\right)}\right)\right) \frac{1-e^{-r H}}{1-e^{-r \frac{H}{m N}}}\right] .
\end{aligned}
$$

\subsubsection{Supplier's discounted cost function}

At each supplier's inventory cycle, the supplier first lasts $T_{q}$ for producing and sending the delivery quantity $q$. After this time, the supplier makes $N-1$ shipments of $q$ units with span length $H /(m N)$ over entire cycle time $H / m$. In each of these cycles, the supplier first produces final products and makes shipments during period $T_{Q}$. Then, the supplier only stocks final products while making deliveries (non- 
producing time). Between two successive deliveries during the production time, the inventory increases with a rate of $P-\theta_{S} I_{S}(t)$, and between two successive shipments during the non-production time, the inventory decreases continuously with a rate of $\theta_{S} I_{S}(t)$ from the $Q_{S}$ level. With all of this in mind, consider the variation of the inventory over the first cycle. Let $N_{Q}=\left\lceil\left(T_{Q}-T_{q}\right) / T_{d}\right\rceil$ be the first delivery during the non-production period, and $T_{i}=i T_{d}+T_{q}$ be the ith-delivery time, where $i=\left\lfloor\left(t-T_{q}\right) / T_{d}\right\rfloor$. The variation of the inventory with respect to time $t, I_{s}(t)$, is governed by the following equations:

For $0 \leq t \leq T_{q}$,

$$
I_{S 1}(t)=\frac{P}{\theta_{S}}\left(1-e^{-\theta_{S} t}\right) \text {. }
$$

For $T_{q} \leq t \leq T_{Q}$

$$
I_{S 2}(t)=\frac{P}{\theta_{S}}\left(1-e^{-\theta_{S}\left(t-T_{i}\right)}\right)+\left(\frac{P}{\theta_{S}}\left(1-e^{-i \theta_{S} T_{d}}\right)-q\left(\sum_{J=0}^{i-1} e^{-J \theta_{S} T_{d}}\right)\right) e^{-\theta_{S}\left(t-T_{i}\right)} .
$$

For $T_{Q} \leq t \leq N_{Q} T_{d}+T_{q}$

$$
I_{S 3}(t)=e^{-\theta_{S}\left(t-T_{Q}\right)} Q_{S} \text {. }
$$

For $N_{Q} T_{d}+T_{q} \leq t \leq(N-1) T_{d}+T_{q}$,

$$
I_{S 4}(t)=e^{-\theta_{S}\left(t-T_{i}\right)}\left(e^{-\theta_{S}\left(T_{i}-T_{Q}\right)} Q_{S}-q \sum_{J=0}^{i-N_{Q}} e^{-J \theta_{S} T_{d}}\right) .
$$

Because at point $t=(N-1) T_{d}+T_{q}$ the supplier's inventory drops to zero, we can obtain $Q_{s}$ from Eq. (10) as

$$
Q_{S}=\frac{q \sum_{J=1}^{N-N_{Q}} e^{-(J-1) \theta_{S} T_{d}}}{e^{-\theta_{S}\left((N-1) T_{d}+T_{q}-T_{Q}\right)}}
$$

After obtaining $Q_{S}$ from Eq. (11), we can then derive $T_{Q}$ using Eq. (8) with the boundary condition $I_{S}\left(T_{Q}\right)=Q s$. Substituting Eq. (11) into this equation and solving for $T_{Q}$ (see Appendix A), we determine that production time $T_{Q}$ is

$$
T_{Q}=\frac{1}{\theta_{S}} L N\left[\frac{\theta_{S} q}{P} e^{\theta_{S}\left((N-1) T_{d}+T_{q}\right)}\left(\sum_{J=1}^{N-1} e^{-(J-1) \theta_{S} T_{d}}\right)+e^{\theta_{S} T_{q}}\right]
$$

Because $T_{Q}$ can be obtained using Eq. (12), we can express the area under the supplier's inventory for the first inventory cycle as

$$
\begin{gathered}
\int_{0}^{T} I_{S}(t)=\int_{0}^{T_{q}} I_{s 1}(t) e^{-r t} d t+\sum_{i=0}^{N_{Q}-2} \int_{T_{i}}^{T_{i+1}} I_{s 2}(t) e^{-r t} d t+\int_{T_{N_{Q}-1}}^{T_{Q}} I_{s 2}(t) e^{-r t} d t \\
+\int_{T_{Q}}^{N_{Q} T_{d}+T_{q}} I_{S 3}(t) e^{-r t} d t+\sum_{i=N_{Q}}^{N-2} \int_{T_{i}}^{T_{i+1}} I_{s 4}(t) e^{-r t} d t .
\end{gathered}
$$


The solution of Eq. (13) is provided by Eq. (B.1) in Appendix B. Hereafter this area will be referred to $\Delta_{S}$. Hence, the present value of the holding costs and disposal costs during entire time horizon $H$, denoted by $P V_{H C_{S}}$, is

$$
P V_{H C_{S}}=P V_{H_{S}}+P V_{C_{S}}=\sum_{i=0}^{m-1}\left(H_{S}+C_{S} \theta_{S}\right) \Delta_{S} e^{-r\left(T_{i}\right)}=\left(H_{S}+C_{S} \theta_{S}\right) \Delta_{S} \frac{1-e^{-r H}}{1-e^{-r \frac{H}{m}}}
$$

and the present value of setup $\operatorname{cost} C$, because there are $m$ setups in the entire time horizon, is

$$
P V_{C}=C \sum_{i=1}^{m} e^{-r\left(T_{i-1}\right)}=C \frac{1-e^{-r H}}{1-e^{-r \frac{H}{m}}}
$$

Thus, the present value of the supplier's total cost is

$$
Z_{S}(m, N)=P V_{H C_{S}}+P V_{C}=\left(C+\left(H_{S}+C_{S} \theta_{S}\right) \Delta_{S}\right) \frac{1-e^{-r H}}{1-e^{-r \frac{H}{m}}}
$$

\subsection{Integrated discounted profit function}

Regardless of whether the aim of using the proposed JIT inventory model is to evaluate or implement a JIT agreement, it is important in this phase to share cost information. Assuming that this requirement has been accomplished successfully, we can derive the integrated discounted total profit function. This function, denoted by $Z_{T}$, includes the present value of sales revenue, and the present value of the costs of both the supplier and buyer.

The present value of the sales revenue is given by

$$
P V_{S R}=s \int_{T_{q}+\tau}^{T_{q}+\tau+H} D e^{-r t} d t=D s \frac{e^{-r\left(T_{q}+\tau\right)}\left(1-e^{-r H}\right)}{r} .
$$

Additionally, the IDP during planning period $H$, including the investment for the JIT alliance $Z_{0}$ is

$$
\begin{aligned}
Z_{T}(m, N) & =e^{-r\left(T_{q}+\tau\right)}\left[D s \frac{1-e^{-r H}}{r}-\left(A+C+\left(H_{S}+C_{S} \theta_{S}\right) \Delta_{S}\right) \frac{1-e^{-r H}}{1-e^{-r \frac{H}{m}}}-\right. \\
& \left.\left(F+q(V+u)+\left(H_{B}+C_{B} \theta_{B}\right) D\left(\frac{e^{\theta_{B} \frac{H}{m N}}-1}{\theta_{B}\left(r+\theta_{B}\right)}+\frac{e^{-\frac{-r H}{m N}}-1}{r\left(r+\theta_{B}\right)}\right)\right) \frac{1-e^{-r H}}{1-e^{\frac{-r H}{m N}}}\right]-Z_{0}
\end{aligned}
$$

Therefore, our problem can be formulated as

maximize $Z_{T}(m, N)$

subject to $N \geq 1, m>m_{1}$.

Note that from Eq. (19), we have not yet specified the lower bound $m_{1}$. Toward this end, consider the buyer's inventory that can be consumed in time length $T_{m}$. If Assumption (iii) holds, then $q=D / \theta$ ${ }_{B}\left(e^{\theta_{B} H /(m N)}-1\right)<P / \theta_{S}\left(1-e^{-\theta_{S} T_{m}}\right)$, hereafter referred to as (I-19), must hold for any $m$ and $N$. Because it is clear that $N \geq 1$, there exists an $m_{1} \geq 1$ for which (I-19) holds. Thus, to determine $m_{1}$, we 
can either vary $m$ from one until (I-19) holds or choose $\left[H / T_{m}\right]$ when substituting $H /(m N)$ for $T_{m}$ in (I19) and numerically solve for $T_{m}$.

\section{Solution procedures}

In this section, we provide some alternatives for solving the model of the previous section. Although there are several ways to face this optimization problem, we discuss those that could be easily implemented in practice. Before doing so, however, it is important to mention here that, it does not seem easy to prove that there cannot exist more than one local minima by using the analytical expressions of the previous section. Consequently, it seems necessary to use an appropriate search routine to find the optimal values of the proposed model. The following method, thus, determines a local minimum but does not provide any guarantee that the obtained minimum is the global minimum.

\subsection{Method I: restricted brute force}

Although the optimization problem in Eq. (19) does not have an upper bound for $m$ and $N$, in most cases, in practice, it is completely reasonable to assume that there exists a lower bound for the time between deliveries (minTd) and an upper bound for the cycle time length (MaxT). Thus, we can determine a very good solution, if not the optimal solution, for integrated discounted profit function $Z_{T}(m, N)$ by evaluating all the combinations that result from varying $m$ and $N$ within $\lceil H / \max T\rfloor \leq m \leq\lfloor H / \operatorname{minT} d\rfloor$ and $1 \leq$ $N \leq\lfloor\max T /(\min T d\lceil H / \max T\rceil)\rfloor$, respectively.

Alternatively, observing that optimizing Eq. (19) with $r \approx 0$ and $H=1$ is equivalent to optimizing the JIT inventory model without the TVM introduced by Yan et al. (2011), it is reasonable to use their upper boundaries. Thus, the upper bounds in Yan et al.'s model are

$$
N \leq N_{U}=\frac{H_{B}+C_{B} \theta_{B}+\theta_{B} V}{H_{S}+C_{S} \theta_{S}} \frac{A+C}{F}+\sqrt{\frac{2 D\left(H_{B}+C_{B} \theta_{B}+\theta_{B} V+\left(\theta_{B} A+\theta_{S} C\right) P\right)}{\left(H_{S}+C_{S} \theta_{S}\right)(P-D)}}
$$

and

$$
q \leq q_{U}=\sqrt{\frac{2 D P(A+C+F)}{P\left(H_{B}+C_{B} \theta_{B}\right)+\left(H_{S}+C_{S} \theta_{S}\right)+C_{B} V P}} .
$$

Hence, considering that solving Eq. (3) for $m$ leads to

$$
m=H \theta_{B} /\left(\ln \left(q \theta_{B} / D+1\right) N\right),
$$

the corresponding boundaries for Eq. (19) are

$$
\begin{aligned}
& 1 \leq N \leq N_{U} \\
& \text { and } \\
& m_{1} \leq m \leq m_{U}=\left\lceil H \theta_{B} /\left(\ln \left(q_{U} \theta_{B} / D+1\right) N\right)\right\rceil,
\end{aligned}
$$

where $m_{U}$ is obtained by replacing $q$ by $q_{U}$ in Eq. (22) and rounding to the closest maximum integer.

\subsection{Method II: using derivatives}

From Eq. (3), if we replace $m$ by $H \theta_{B} /\left(\ln \left(q \theta_{B} / D+1\right) N\right)$ in Eq. (18), then we obtain the IDP as a function of $q, N$. With this new function, we can then consider $N$ as a constant, and use the first-order necessary condition for optimality. Thus, by letting $Z=Z_{T}(q, N)$ be this resulting function, the solution procedure using derivatives is as follows:

Step 1: Select a plausible range of values for $\left(N_{\min } \leq N \leq N_{\max }\right)$, as described in Method I. 
Step 2: Use any initial estimates of $N$ and $m$. Let $\vec{X}=\left\{m_{0}, N_{0}\right\}$ be the best current solution.

Step 3: Let $N_{t}=N_{0}$, and derive the partial derivatives of $Z$. Then use the feasible interval for $q, 0<$ $q<\left(e^{\theta_{B} H / m_{1}}-1\right) D / \theta_{B}$, to determine all the points that satisfy $\partial Z / \partial q=0$. From these points, denote the best for $Z$ as $\vec{X}_{T}=\left\{q_{t}, N_{t}\right\}$.

Step 4: Evaluate and select a solution. If $Z\left(\vec{X}_{T}\right)>Z(\vec{X})$, then $\vec{X}=\vec{X}_{T}$. If $N=N_{\text {max }}$, then go to Step 5; otherwise, set $N_{0}=N_{0}+1$ and repeat Steps 3-4.

Step 5: With $\left\{q_{c}, N_{c}\right\}$ as the current $\vec{X}$, compute $m_{c}=H \theta_{B} /\left(\ln \left(q_{c} \theta_{B} / D+1\right) N_{c}\right)$. If $Z_{T}\left(\left\lceil m_{c}\right\rceil, N_{c}\right)>$ $Z_{T}\left(\left\lfloor m_{c}\right\rfloor, N_{c}\right)$, then use $\left\{\left\lceil m_{c}\right\rfloor, q_{c}\right\}$ as the best solution; otherwise, use $\left\{\left\lfloor m_{c}\right\rfloor, q_{c}\right\}$.

\subsection{Method III: use optimization software}

Several options are available to address the optimization problem given in Eq. (19). However, in this paper, we test the differential evolution method incorporated in Mathematica software with a scaling factor of 0.6 and maximum number of iterations of 500. These parameters were chosen subjectively to obtain the best performance for the method.

\subsection{Method IV: using a cost function that neglects the TVM}

An interesting alternative that may arise for solving the optimization problem in Eq. (19) includes using of the integrated inventory cost function that Yan et al. (2011), Sarkar (2013), and Chang (2014) considered. Although this cost function neglects the effect of the TVM, as we shall see later, the optimal solution of this function can provide a very good approximation for solving Eq. (19). Following on from this idea, the total cost function to use, including unit cost $u$, is

$$
\begin{aligned}
T C(q, N)=\left(\frac{D}{N q}+\frac{\theta_{B}}{2 N}\right)(A+C+N F+(V+u) N q) \\
+\frac{q}{2}\left[\left(H_{B}+C_{B} \theta_{B}\right)+\left(H_{S}+C_{S} \theta_{S}\right)\left\{\frac{(2-N) D}{P}+N-1\right\}\right]
\end{aligned}
$$

and the steps to be performed are as follows:

Step 1: Let Eq. (25) be $Z$ and execute Steps 1-4 of method II.

Step 2: With $\left\{q_{c}, N_{c}\right\}$ as the best current solution, use Eq. (22) to obtain $m_{c}$; that is, compute $m_{c}=H \theta_{B} /$ $\left(\ln \left(q_{c} \theta_{B} / D+1\right) N_{c}\right)$.

Step 3: Select two positive integers, $\Delta_{m} \geq 0$ and $\Delta_{N} \geq 0$, to perform a local search around $\left\{m_{c}, N_{c}\right\}$. For all the feasible integers within the intervals; $\left\lfloor m_{c}\right\rfloor-\Delta_{m} \leq m_{c} \leq\left\lceil m_{c}\right\rceil+\Delta_{m}$ and $N_{c} \leq N_{c}+\Delta_{N}$, use Eq. (18) to calculate the corresponding IDP values.

Step 4: Choose the $m$ and $N$ pair that results in the maximum IDP in Step 3.

\subsection{Method V: using Eq. (25) without derivatives}

Instead of using the first-order necessary conditions required for Methods II and IV, we can take advantage of the improved solution procedure proposed by Chang (2014) to optimize Eq. (25). By doing this, we simply have to optimize Eq. (25) using through Chang's procedure and then follow Steps 2-4 of Method IV.

\section{Results and Discussion}

We consider two examples that are extended versions of the illustrations provided by Kim and Ha (2003), Yan et al. (2011), and Sarkar (2013). The first example is used to analyze the effect of the TVM on inventory policies and the second example is used to compare the solution procedures described in Section 4. 
Example 1. To analyze the effect of the TVM on the modeling of JIT inventory systems, consider the numerical example presented in Yan et al. (2011), where $A=25, C=600, C_{b}=C_{s}=50, D=4800, F=$ $50, H_{B}=7, H_{S}=6, P=19200, V=1$, and $\theta_{B}=\theta_{S}=0.05$. To complement these data, also consider $\tau=$ $1 / 360, u=7 / 0.16, s=1.12 \cdot u, H=5$, and $Z_{0}=28930$. Based on these data, Table 1 shows the solutions provided by Method I for varying discount rate $r$ from $2.5 \%$ to $52.5 \%$. According to the table, the IDP decreased with increasing $r$. This was not unexpected because the discount rate represents a minimum rate of return desired or acceptable to the decision maker; thus, the higher this rate, the lower the present value of each future cash flow.

Regarding the effect of the discount rate on the decision variables, it is interesting to note that an increase in $r$ tends to reduce delivery lot-size $q$, but almost without impacting $m$ and, thus, $T$ and $Q_{P}$. i.e., primarily through the number of deliveries per production batch, $N$. As a result, it appears that increasing the number of deliveries mitigates the negative effect of a higher discount rate.

Another notable result, suggested by Table 1, is that the optimal inventory policy when accounting with the TVM seems to be close to that when neglecting the TVM. In particular, for this example, optimizing Eq. (25) lead to $\{q=244.9, N=4\}$, which, for the planning period of $H=5$, implies choosing between $\{m=24, N=4\}$ and $\{m=25, N=4\}$ : a maximum difference for $N$ of 4 and for $m$ of 1 . In fact, Method IV and V exploit this when attempting to determine the optimal solution of Eq. (19).

Table 1

Summary of results, $\left\{I D P, m, N, q, T, Q_{p}\right\}$, for different $r$ values.

\begin{tabular}{|c|c|c|c|c|c|c|c|c|c|c|c|}
\hline \multirow[b]{2}{*}{ Variable } & \multicolumn{11}{|c|}{$\mathrm{r}$} \\
\hline & 0.025 & 0.075 & 0.125 & 0.175 & 0.225 & 0.275 & 0.325 & 0.375 & 0.425 & 0.475 & 0.525 \\
\hline$I D P$ & 28212 & 20573 & 14287 & 9001 & 4600 & 854 & -2331 & -5039 & -7381 & -9407 & -11153 \\
\hline$m$ & 25 & 24 & 25 & 24 & 25 & 25 & 24 & 25 & 25 & 24 & 25 \\
\hline$N$ & 4 & 5 & 5 & 6 & 6 & 6 & 7 & 7 & 7 & 8 & 8 \\
\hline$q$ & 240 & 200 & 192 & 167 & 160 & 160 & 143 & 137 & 137 & 125 & 120 \\
\hline$T$ & 0.20 & 0.21 & 0.20 & 0.21 & 0.20 & 0.20 & 0.21 & 0.20 & 0.20 & 0.21 & 0.20 \\
\hline$Q_{P}$ & 964 & 1004 & 964 & 1004 & 964 & 964 & 1004 & 964 & 964 & 1004 & 964 \\
\hline
\end{tabular}

Although the closeness of the suggested solution of Eqs. (19) and (24) may entice decision makers to use Eq. (24) instead of Eq. (19) because of its simplicity, caution should be taken when using this approach indiscriminately because it can be misleading. For example, suppose that we are interested in evaluating the viability of adopting a JIT purchasing system under a minimum attractive rate of return of 0.275 . If $Z_{0}$ which represents the investment of that JIT alliance, ranges between any value greater than 28929.30, then both of the solutions suggested by Eq. (24), $\{m=24, N=4\}$ and $\{m=25, N=4\}$, lead to rejecting the JIT partnership. By contrast, as can be verified from Table 1, the JIT partnership is feasible with $\{m=24, N=7\}$ for any value less than or equal to 29784 (i.e., $\forall Z_{0} \leq 28930-854=29784$ ). As a result, we note that, for any $Z_{o}$ between 28929.30 and 29784, the decision would be to reject the JIT purchasing system when it is in fact viable.

Finally, to provide additional insight, Fig. 2 shows the impact of deterioration on $m, Q_{P}, q$, and $N$ by varying deterioration between 0.025 and 0.2 . As can be observed, when deterioration increases, $Q_{p}$ and $q$ decrease without $N$ changing for the purpose of benefiting the JIT partnership. Indeed, this relationship was also noted by Yan et al. (2011) for Eq. (24). In terms of optimizing Eq. (19), the reduction of $Q_{p}$ and $q$ is the consequence of increasing $m$ while not changing $N$. Additionally, note that this pattern is in line with typical JIT programs, where the reduction of setup times implies more dedication of the supplier to the JIT partnership (a greater cycle time and a greater production lot-size) together with the reduction of product deterioration in the entire supply chain. 

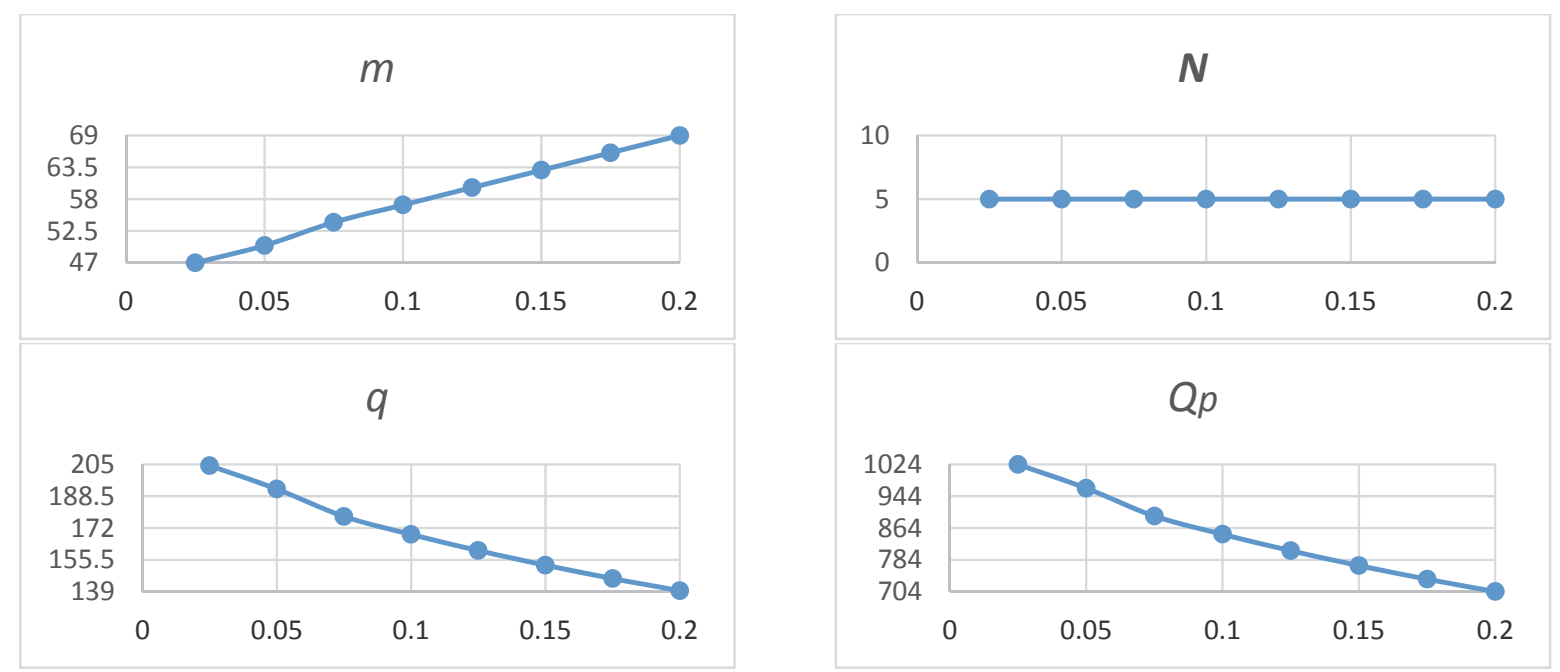

Fig. 2. Variation of the optimal values $m, N, q$, and $Q$ with respect to deterioration

Example 2. To compare the performance of the solution procedures outlined in the previous section, we first consider the following data: $A=25, C=600, C_{b}=C_{S}=50, D=4800, F=50, H=10, H_{B}=$ $7, H_{s}=6, P=10000, r=14 \%, s=51.63, \tau=1 / 365, u=43.75, V=1, \theta_{B}=\theta_{S}=0.2$ and $Z_{o}=$ 0 . For these data, the optimal solution is $\{I D P=117976, m=57, N=7\}$, which implies that $q=$ 120.603 and $T=0.175439$ (see Fig. 3). However, when $F=1800$, the optimal solution is $\{I D P=$ 10466.8, $m=64, N=1$, which implies that $q=761.842$ and $T=0.15625$. As can be observed in Table 2, for the former case, all the methods in consideration, with the exception of Methods IV and V (under $\Delta_{m}=\Delta_{N}=0$ ), can determine the optimal solution. For the latter case, however, we note that Method III only determines the optimal solution when we make the search interval narrower, and Methods IV and V provide an IPD that is now $6 \%$ lower than that of the optimal value. Regarding the time length consumed by these methods, we observe that the best performance time is achieved by Method IV, followed by Methods III, II, and I. As a result, the main conclusions that we draw are as follows:

- Method IV and V are significantly faster than the other methods, but may only determine near optimal solutions when setting the method up for achieving its maximum speed.

- Method III is still significantly faster than the other methods, but in some scenarios, it may only determine the optimal solution within a specified search interval.

- Methods I and II can determine the optimal solution for a given range of $N$. However, Method I can be significantly slower than Method II when searching in a wide range of $m$.

Table 2

Performance of the methods under 20 replicates, $1 \leq N \leq 60$ for Methods I-IV.

\begin{tabular}{|c|c|c|c|c|c|}
\hline \multirow{2}{*}{ Method* } & \multirow{2}{*}{$\begin{array}{l}\text { Search interval } \\
\quad \text { for } m\end{array}$} & \multicolumn{2}{|c|}{ Example 1 with $F=50$} & \multicolumn{2}{|c|}{ Example 1 with $F=1800$} \\
\hline & & Optimal? & $\begin{array}{c}\text { Average time } \\
\text { (seconds) }\end{array}$ & Optimal? & $\begin{array}{c}\text { Average time } \\
\text { (seconds) }\end{array}$ \\
\hline $\mathrm{I}$ & {$[30,1800]$} & Yes & 43.051 & Yes & 43.1231 \\
\hline II & $m \geq 3$ & Yes & 27.8329 & Yes & 20.1284 \\
\hline III & $m \geq 3$ & Yes & 4.01661 & No: $\{6709,38,2\}$ & 3.89814 \\
\hline III & {$[30,1800]$} & Yes & 3.96725 & Yes & 3.56932 \\
\hline IV & Best closer & No: $\{117788,58,6\}$ & 0.0440038 & No: $\{9841,59,1\}$ & 0.037422 \\
\hline $\mathrm{V}$ & integers & No: $\{117788,58,6\}$ & 0.0149414 & No: $\{9841,59,1\}$ & 0.00792864 \\
\hline
\end{tabular}


Although the performance average time may seem low for all these methods, we should be aware that, in practice, an inventory system controls thousands of distinct type of items or stock keeping units (SKUs). Hence, an optimal inventory policy must be estimated not only for each of these SKUs but also for each of the subsystems within the multi-echelon inventory system. For example, if Method I (or II) is applied to define the optimal inventory control levels of a retailer that manages 400 stores with 1000 SKUs each, then we would wait approximately 199 days (or 129) to determine these control levels.

Despite this difficulty, we can improve the performance of Method I (or II) by assuming that the IDP provided by Eq. (18) is a discrete concave function within a selected region of interest. As shown in Fig. 3 , it is not necessary to evaluate the entire range of $m$ and $N$ (or $N$ ) in such circumstances. Instead, for each $N$, we can stop searching for the best $m$ as soon as Eq. (18) starts to decrease, and also, for each point of $N$ and best $m$ found, we can stop searching for the best solution as soon as the evaluated function no longer improves. By doing this in Example 1 with $F=50$, the average time for Method I is 1.97897 and 1.03166 for Method II, a reduction of $95.4 \%$ and $96.3 \%$ over the corresponding average times reported in Table 2. If, however, a conservative approach is preferred, then a possible option to improve not only Methods I and II but Methods III and IV can result in using Eqs. (23) and (24) as search intervals for $m$ and $N$, for which the values in Table 2 would be $1 \leq N \leq 25$ for Methods I-IV, $111 \leq m \leq 1800$ for Methods I and III, and $m \geq 111$ for Method II.

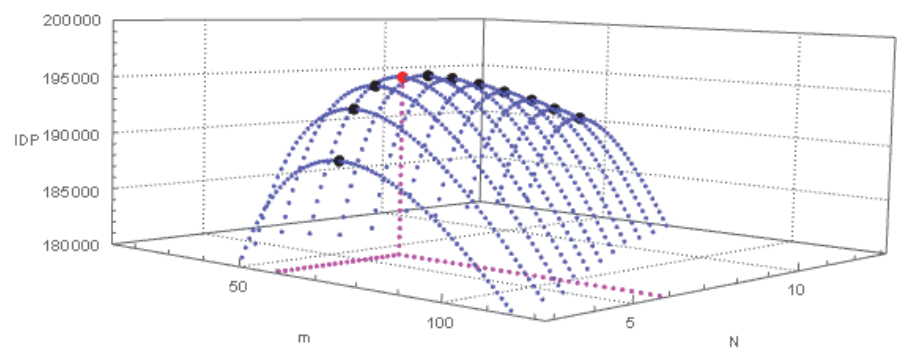

Fig. 3. IDP as a function of $m$ and $N$ (Example 1)

Regarding Methods IV and V, it is worth noting that the optimal solution would have been found if we had extended the search of $m$ and $N$ to more than the two closest integers of $\left\{m_{c}, N_{c}\right\}$ in Step 2. Specifically, because Step 2 of Methods IV and V leads to $\left\{m_{c}=57.20, N=6\right\}$ when $F=50$, and to $\left\{m_{c}=58.7, N=1\right\}$ when $F=1800$, these methods can determine the optimal solution whenever the local search in Step 3 is performed under any $\left\{\Delta_{m} \geq 0, \Delta_{N} \geq 1\right\}$ in the former case, and $\left\{\Delta_{m} \geq 4, \Delta_{N} \geq\right.$ $0\}$ in the latter case. From our computational experience, we note that it is sufficient to select $\left\{\Delta_{m}=10\right.$, $\left.\Delta_{N}=6\right\}$. By selecting these step sizes, the average performance time in seconds for Method IV and V are $\{0.9365,0.766\}$ and $\{0.9062,0.7485\}$, respectively, which still outperforms the other methods.

\section{Conclusions}

In this article, we have analyzed JIT purchasing agreements under the TVM by proposing a new inventory model that extended the integrated multi-lot-size production-inventory model for deteriorating items proposed by Yan et al. (2011). Through the effort of gaining an understanding of the TVM effect over the JIT lot sizing calculation, we have used differential calculus to derive cost functions instead of the algebraic approach used by Yan et al. (2011). Furthermore, because providing exact expression for optimality was found to be very difficult, we have provided and analyzed five numerical methods that can be easily implemented in practice. The first consisted of an exhaustive search. The second used derivative information from our derived model, Eq. (19). The third used optimization software, and the fourth and five performed a local search from the optimal solution obtained through Yan et al.' model: the fourth using derivative information and the fifth using the solution procedure proposed by Chang (2014). In general, our results suggest that, for the sake of benefiting the entire supply chain, the presence of a discount rate on lot-size calculation had significantly more impact on the delivery lot-size than the 
production lot-size by primarily adjusting the number of deliveries per production batch (see Table 1). This result is different from what occurs to counterbalance the presence of a higher deterioration rate, where both the production lot-size and delivery lot-size are reduced without changing the number of deliveries (see Fig. 2). Another notable result, suggested by Table 1 and Table 2, is that Yan et al.'s model generates near-optimal solutions for our derived model. This property, together with our findings regarding the effect of the discount rate on lot-size calculation, was exploited by Methods IV and V. Our results, in this regard, demonstrate that these two methods are significantly more effective than the other alternatives analized. See Example 2 for a complete discussion of these approaches.

Although from our computational experience, we can attest that Eq. (18) is a discrete concave function and, thus, all the above results can be generalized, the findings of this study are restricted to our numerical experiments. Overall, the convenience of our approach mainly depends on how sensitive the IDP is to changes in the decision variable, but even in the presence of low sensitivity, using our proposed model can be decisive in terms of promoting or rejecting a JIT partnership. Under low sensitivity, one valid approach for evaluating the attractiveness of long-term JIT partnerships may involve the use of a discounted cash flow with an inventory model that neglects the TVM, that is, a different inventory policy is determined using yearly estimated costs through Yan et al.'s model. However, from Example 1, we found that this methodology could be misleading. In this matter, one interesting contribution of future applied studies may encompass the convenience of our approach over different discounted cash flow analysis. Finally, our proposed model can be further improved by including additional inventory system features. Thus, some potential topics for future research include the modeling of multi-echelon systems, complementary and substitute multi-items, and pricing strategies.

\section{Acknowledgement}

We thank Maxine Garcia, PhD, from Edanz Group (www.edanzediting.com/ac) for editing a draft of this manuscript.

\section{References}

Aderohunmu, R., Mobolurin, A., \& Bryson, N. (1995). Joint vendor-buyer policy in JIT manufacturing. Journal of The Operational Research Society, 46(3), 375-385.

Ali, A., Santini, N., \& Rahman, M. A. (2012). Kanban supplier system as a standardisation method and WIP reduction. International Journal of Industrial and Systems Engineering, 11(1-2), 179-188.

Andriolo, A., Battini, D., Grubbström, R. W., Persona, A., \& Sgarbossa, F. (2014). A century of evolution from Harris's basic lot size model: Survey and research agenda. International Journal of Production Economics, 155(Supplement C), 16-38.

Banerjee, A., \& Kim, S.-L. (1995). An integrated JIT inventory model. International Journal of Operations \& Production Management, 15(9), 237.

Chang, H.-C. (2014). An analysis of production-inventory models with deteriorating items in a two-echelon supply chain. Applied Mathematical Modelling, 38(3), 1187-1191.

Chen, Z.-X., \& Tan, K. H. (2011). The perceived impact of JIT implementation on operations performance. Journal of Advances in Management Research, 8(2), 213-235.

Chen, Z., \& Tan, K. H. (2013). The impact of organization ownership structure on JIT implementation and production operations performance. International Journal of Operations \& Production Management, 33(9), 1202-1229.

Chiarini, A. (2017). An adaptation of the EOQ formula for JIT quasi-pull system production. Production Planning \& Control, 28(2), 123-130.

Darlington, J., Francis, M., Found, P., \& Thomas, A. (2016). Targeting lean process improvement projects for maximum financial impact. Production Planning \& Control, 27(2), 114-132.

Ha, D., \& Kim, S.-L. (1997). Implementation of JIT purchasing: An integrated approach. Production Planning \& Control, 8(2), 152-157.

Joglekar, P. N. (1988). Comments on "A quantity discount pricing model to increase vendor profits". Management Science, 34(11), 1391-1398. 
Kim, S.-L., \& Ha, D. (2003). A JIT lot-splitting model for supply chain management: Enhancing buyer-supplier linkage. International Journal of Production Economics, 86(1), 1-10.

Larson, P. D. (1989). An Inventory Model Which Assumes The Problem Away: A Note O. Production and Inventory Management Journal, 30(4), 73.

Lin, Y. S., Yu, J. C. P., \& Wang, K.-J. (2009). An efficient replenishment model of deteriorating items for a supplier-buyer partnership in hi-tech industry. Production Planning \& Control, 20(5), 431-444.

Matsui, Y. (2007). An empirical analysis of just-in-time production in Japanese manufacturing companies. International Journal of Production Economics, 108(1), 153-164.

Michelsen, C. J., O'Connor, P., \& Wiseman, T. (2014). Just in Time. Defense AT\&L, 43(2), 32-36.

Negrão, L. L. L., Godinho Filho, M., \& Marodin, G. (2017). Lean practices and their effect on performance: a literature review. Production Planning \& Control, 28(1), 33-56.

Pan, A. C., \& Liao, C.-J. (1989). An Inventory Model Under Just-In-Time Purchasing Agreement. Production and Inventory Management Journal, 30(1), 49.

Ramasesh, R. V. (1990). Recasting the Traditional Inventory Model to Implement Just-in-Time Purchasing. Production and Inventory Management Journal, 31(1), 71.

Rau, H., Wu, M.-Y., \& Wee, H.-M. (2003). Integrated inventory model for deteriorating items under a multiechelon supply chain environment. International Journal of Production Economics, 86(2), 155-168.

Santos, J., Wysk, R., Manuel Torres, J., Santos, J., Wysk, R., \& Manuel Torres, J. (2006). Continuous Improvement Tools Improving Production with Lean Thinking (pp. 1-17): John Wiley \& Sons, Inc.

Sarkar, B. (2013). A production-inventory model with probabilistic deterioration in two-echelon supply chain management. Applied Mathematical Modelling, 37(5), 3138-3151.

White, R. E., Pearson, J. N., \& Wilson, J. R. (1999). JIT Manufacturing: A Survey of Implementations in Small and Large U.S. Manufacturers. Management Science, 45(1), 1-15. doi:10.1287/mnsc.45.1.1

Xu, Y., \& Chen, M. (2016). Improving Just-in-Time Manufacturing Operations by Using Internet of Things Based Solutions. Procedia CIRP, 56(Supplement C), 326-331.

Yan, C., Banerjee, A., \& Yang, L. (2011). An integrated production-distribution model for a deteriorating inventory item. International Journal of Production Economics, 133(1), 228-232.

\section{Appendix A: Derivation of $\boldsymbol{T}_{Q}$}

Let $T_{Q}^{\prime}=\left(N_{Q}-1\right) H /(m N)+T_{q}$. Because at time $T_{Q}$ the producer's inventory level is $I_{S 2}\left(T_{Q}\right)=Q_{S}$, from Eqs. (8) and (11), we have

$$
\begin{aligned}
& \frac{P}{\theta_{S}}\left(1-e^{-\theta_{S}\left(T_{Q}-T_{Q}^{\prime}\right)}\right)+\left(\frac{P}{\theta_{S}}\left(1-e^{-\left(N_{Q}-1\right) \theta_{s} T_{d}}\right)-q\left(\sum_{J=0}^{N_{Q}-2} e^{-J \theta_{s} T_{d}}\right)\right) e^{-\theta_{S}\left(T_{Q}-T_{Q}^{\prime}\right)}=\frac{q \sum_{J=1}^{N-N_{Q}} e^{-(J-1) \theta_{S} T_{d}}}{e^{-\theta_{S}\left((N-1) T_{d}+T_{q}-T_{Q}\right)}} \\
& \Rightarrow \frac{P}{\theta_{S}}\left(1-e^{-\theta_{S}\left(T_{Q}-T_{Q}^{\prime}\right)}\right) \boldsymbol{e}^{\boldsymbol{\theta}_{s} T_{Q}}+\boldsymbol{e}^{\boldsymbol{\theta}_{s} T_{Q}}\left(\frac{P}{\theta_{S}}\left(1-e^{-\left(N_{Q}-1\right) \theta_{S} T_{d}}\right)-q\left(\sum_{J=0}^{N_{Q}-2} e^{-J \theta_{S} T_{d}}\right)\right) e^{-\theta_{S}\left(T_{Q}-T_{Q}^{\prime}\right)}=\frac{q \sum_{J=1}^{N-N_{Q}} e^{-(J-1) \theta_{s} T_{d}}}{e^{-\theta_{S}\left((N-1) T_{d}+T_{q}\right)}} \\
& \Rightarrow \frac{P}{\theta_{S}}\left(\boldsymbol{e}^{\boldsymbol{\theta}_{S} T_{Q}}-\boldsymbol{e}^{\boldsymbol{\theta}_{s} T_{Q}^{\prime}}\right)+\left(\frac{P}{\theta_{S}}\left(1-e^{-\left(N_{Q}-1\right) \theta_{S} T_{d}}\right)-q\left(\sum_{J=0}^{N_{Q}-2} e^{-J \theta_{s} T_{d}}\right)\right) \boldsymbol{e}^{\boldsymbol{\theta}_{s} T_{Q}^{\prime}}=\frac{q \sum_{J=1}^{N-N_{Q}} e^{-(J-1) \theta_{S} T_{d}}}{e^{-\theta_{S}\left((N-1) T_{d}+T_{q}\right)}} \\
& \Rightarrow \frac{\boldsymbol{P}}{\boldsymbol{\theta}_{s}} \boldsymbol{e}^{\boldsymbol{\theta}_{s} T_{Q}}=\frac{q \sum_{J=1}^{N-N_{Q}} e^{-(J-1) \theta_{s} T_{d}}}{e^{-\theta_{S}\left((N-1) T_{d}+T_{q}\right)}}-\left(\frac{P}{\theta_{s}}\left(1-e^{-\left(N_{Q}-1\right) \theta_{s} T_{d}}\right)-q\left(\sum_{J=0}^{N_{Q}-2} e^{-J \theta_{s} T_{d}}\right)\right) e^{\theta_{s} T_{Q}^{\prime}}+\frac{P}{\theta_{s}} e^{\theta_{s} T_{Q}^{\prime}} \\
& \Rightarrow \boldsymbol{e}^{\theta_{s} T_{Q}}=\frac{\theta_{S} q \sum_{J=1}^{N-N_{Q}} e^{-(J-1) \theta_{s} T_{d}}}{P e^{-\theta_{S}\left((N-1) T_{d}+T_{q}\right)}}-\left(\left(1-e^{-\left(N_{Q}-1\right) \theta_{S} T_{d}}\right)-\frac{\theta_{S} q}{P}\left(\sum_{J=0}^{N_{Q}-2} e^{-J \theta_{S} T_{d}}\right)\right) e^{\theta_{S} T_{Q}^{\prime}}+e^{\theta_{S} T_{Q}^{\prime}} \\
& \Rightarrow T_{Q}=\frac{\mathbf{1}}{\boldsymbol{\theta}_{\boldsymbol{s}}} \boldsymbol{L N}\left[\frac{\theta_{s} q \sum_{J=1}^{N-N_{Q}} e^{-(J-1) \theta_{s} T_{d}}}{P e^{-\theta_{S}\left((N-1) T_{d}+T_{q}\right)}}-\left(\left(1-e^{-\left(N_{Q}-1\right) \theta_{s} T_{d}}\right)-\frac{\theta_{s} q}{P}\left(\sum_{J=0}^{N_{Q}-2} e^{-J \theta_{s} T_{d}}\right)\right) e^{\theta_{S} T_{Q}^{\prime}}+e^{\theta_{s} T_{Q}^{\prime}}\right]
\end{aligned}
$$




$$
\begin{aligned}
& \Rightarrow T_{Q}=\frac{1}{\theta_{S}} L N\left[\frac{\theta_{S} q \sum_{J=1}^{N-N_{Q}} e^{-(J-1) \theta_{s} T_{d}}}{P e^{-\theta_{S}\left((N-1) T_{d}+T_{q}\right)}}+e^{\theta_{s} T_{q}}+\frac{\theta_{S} q}{P} e^{\theta_{s} T_{Q}^{\prime}}\left(\sum_{J=0}^{N_{Q}-2} e^{-J \theta_{s} T_{d}}\right)\right] \\
& \Rightarrow T_{Q}=\frac{1}{\theta_{S}} L N\left[\frac{\theta_{S} q \sum_{J=1}^{N-N_{Q}} e^{-(J-1) \theta_{S} T_{d}}}{P e^{-\theta_{S}\left((N-1) T_{d}+T_{q}\right)}}+e^{\theta_{S} T_{q}}+\frac{\theta_{S} q}{P} e^{\theta_{S} T_{Q}^{\prime}}\left(\sum_{J=1}^{N_{Q}-1} e^{-(J-1) \theta_{S} T_{d}}\right)\right] \\
& \Rightarrow T_{Q}=\frac{1}{\theta_{S}} L N\left[\frac{\theta_{S} q}{P} e^{\theta_{S}\left((N-1) T_{d}+T_{q}\right)}\left(\sum_{J=1}^{N-N_{Q}} e^{-(J-1) \theta_{S} T_{d}}+e^{-\left(N-N_{Q}\right) \theta_{s} T_{d}}\left(\sum_{J=1}^{N_{Q}-1} e^{-(J-1) \theta_{S} T_{d}}\right)\right)+e^{\theta_{S} T_{q}}\right] \\
& \Rightarrow T_{Q}=\frac{1}{\theta_{S}} L N\left[\frac{\theta_{S} q}{P} e^{\theta_{S}\left((N-1) T_{d}+T_{q}\right)}\left(1+e^{-\theta_{s} T_{d}}+\cdots e^{-\left(N-N_{Q}-1\right) \theta_{s} T_{d}}+e^{-\left(N-N_{Q}\right) \theta_{S} T_{d}}+\cdots e^{-(N-2) \theta_{s} T_{d}}\right)+e^{\theta_{S} T_{q}}\right] .
\end{aligned}
$$

Hence,

$T_{Q}=\frac{1}{\theta_{S}} L N\left[\frac{\theta_{S} q}{P} e^{\theta_{S}\left((N-1) T_{d}+T_{q}\right)}\left(\sum_{J=1}^{N-1} e^{-(J-1) \theta_{s} T_{d}}\right)+e^{\theta_{s} T_{q}}\right]$.

Appendix B: Area under the supplier's inventory (first cycle)

Recall that $N_{Q}=\left[\frac{\left(T_{Q}-T_{q}\right)}{H /(m N)}\right\rceil$ and $T_{i}=i \frac{H}{m N}+T_{q}$, where $i \in \mathbb{Z}$. For convenience, we denote the power of $e$ as $\exp [\ldots]$, so from Eq. (13), we know that

$$
\begin{gathered}
\int_{0}^{T} I_{S}(t)=\int_{0}^{T_{q}} I_{s 1}(t) \exp [-r t] d t+\sum_{i=0}^{N_{Q}-2} \int_{T_{i}}^{T_{i+1}} I_{s 2}(t) \exp [-r t] d t+\int_{T_{N_{Q}-1}}^{T_{Q}} I_{s 2}(t) \exp [-r t] d t+ \\
\int_{T_{Q}}^{N_{Q} \frac{H}{m N}+T_{q}} I_{S 3}(t) \exp [-r t] d t+\sum_{i=N_{Q}}^{N-2} \int_{T_{i}}^{T_{i+1}} I_{S 4}(t) \exp [-r t] d t .
\end{gathered}
$$

Hence,

$$
\begin{aligned}
& \Delta_{S}= \int_{0}^{T} I_{S}(t)=\frac{\exp \left[-\left(r+\theta_{S}\right)\left(T_{q}+T_{Q}+\frac{H}{m}\right)\right]}{\left(r+\theta_{S}\right)\left(\exp \left[\theta_{S} \frac{H}{m N}\right]-1\right)\left(\exp \left[\frac{H}{m N}\right]-1\right)}\left(q \left\{\exp \left[\theta_{S}\left(2 T_{q}+(N+1) \frac{H}{m N}\right)+r\left(T_{q}+\frac{H}{m}\right)\right]-\right.\right. \\
& \exp \left[\theta_{S}\left((N+1) \frac{H}{m N}+2 T_{q}\right)+r\left((N+1) \frac{H}{m N}+T_{q}\right)\right]-\exp \left[\theta_{S}\left(\left(N+N_{Q}\right) \frac{H}{m N}+2 T_{q}\right)+\right. \\
& r\left.\left(\frac{H}{m}+T_{q}\right)\right]+\exp \left[\theta_{S}\left(\left(N+N_{Q}\right) \frac{H}{m N}+2 T_{q}\right)+r\left((N+1) \frac{H}{m N}+T_{q}\right)\right]-\exp \left[\theta _ { S } \left(T_{q}+T_{Q}+\right.\right. \\
&\left.\left.(N+1) \frac{H}{m}\right)+r\left(\frac{H}{m}+T_{Q}\right)\right]+\exp \left[\left(\theta_{S}+r\right)\left(T_{Q}+\frac{H}{m}\right)+\theta_{S} T_{q}\right]-\exp \left[\theta_{S}\left(T_{q}+T_{Q}+\frac{H}{m}\right)+\right. \\
&\left.r\left(\frac{H}{m N}+T_{Q}\right)\right]+\exp \left[\theta_{S}\left(T_{q}+T_{Q}+(N+1) \frac{H}{m}\right)+r\left(T_{Q}+2 \frac{H}{m N}\right)\right]+\exp \left[\theta _ { S } \left(T_{q}+T_{Q}+\right.\right. \\
&\left.\left.\left.\left(N_{Q}+1\right) \frac{H}{m N}\right)+r\left(\frac{H}{m N}+T_{Q}\right)\right]-\exp \left[\theta_{S}\left(T_{q}+T_{Q}+\left(N_{Q}+1\right) \frac{H}{m N}\right)+r\left(2 \frac{H}{m N}+T_{Q}\right)\right]\right\}+ \\
& Q_{S}\left\{\exp \left[\left(2 \theta_{S}+r\right)\left(T_{Q}+\frac{H}{m N}\right)\right]+\exp \left[\left(\theta_{S}+r\right)\left(T_{q}+\frac{H}{m}\right)+\theta_{S} T_{Q}\right]+\exp \left[( \theta _ { S } + r ) \left(T_{q}+\right.\right.\right. \\
&\left.\left.(N+1) \frac{H}{m N}\right)+\theta_{S} T_{Q}\right]+\exp \left[\left(\theta_{S}+2 r\right) \frac{H}{m N}+\left(r+2 \theta_{S}\right) T_{Q}\right]-\exp \left[2 \theta_{S}\left(\frac{H}{m N}+T_{Q}\right)+\right. \\
&\left.r\left(2 \frac{H}{m N}+T_{Q}\right)\right]-\exp \left[\theta_{S}\left(T_{q}+T_{Q}+\frac{H}{m}\right)+r\left((N+1) \frac{H}{m N}+T_{q}\right)\right]-\exp \left[\theta _ { S } \left(T_{q}+T_{Q}+\right.\right. \\
&\left.\left.\left.\left.(N+1) \frac{H}{m N}\right)+r\left(T_{q}+\frac{H}{m}\right)\right]-\exp \left[\theta_{S}\left(\frac{H}{m N}+2 T_{Q}\right)+r\left(T_{Q}+\frac{H}{m N}\right)\right]\right\}\right)+\frac{P}{\left(r+\theta_{S}\right) r \theta_{S}}(r(\exp [-(r+ \\
&\left.\left.\left.\left.\quad \theta_{S}\right) T_{q}\right]-\exp \left[-r T_{q}\right]+\exp \left[\theta_{S} T_{q}-\left(\theta_{S}+r\right) T_{Q}\right]-\exp \left[-r T_{Q}\right]\right)+\theta_{S}\left(1-\exp \left[-r T_{Q}\right]\right)\right),
\end{aligned}
$$

where $Q_{s}$ and $T_{Q}$ are given by Eq. (11) and Eq. (12), respectively.

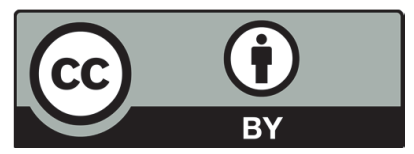

(C) 2019 by the authors; licensee Growing Science, Canada. This is an open access article distributed under the terms and conditions of the Creative Commons Attribution (CCBY) license (http://creativecommons.org/licenses/by/4.0/). 